\title{
Coherent dynamics of Bose-Einstein condensates in high-finesse optical cavities
}

\author{
Peter Horak ${ }^{1}$, Stephen M. Barnett ${ }^{1}$, and Helmut Ritsch ${ }^{2}$ \\ ${ }^{1}$ Department of Physics and Applied Physics, University of Strathclyde, Glasgow G4 ONG, United Kingdom \\ ${ }^{2}$ Institut für Theoretische Physik, Universität Innsbruck, Technikerstr. 25, A-6020 Innsbruck, Austria
}

(August 2, 2006)

We study the mutual interaction of a Bose-Einstein condensed gas with a single mode of a high-finesse optical cavity. We show how the cavity transmission reflects condensate properties and calculate the self-consistent intra-cavity light field and condensate evolution. Solving the coupled condensate-cavity equations we find that while falling through the cavity, the condensate is adiabatically transfered into the ground state of the periodic optical potential. This allows time dependent non-destructive measurements on BoseEinstein condensates with intriguing prospects for subsequent controlled manipulation.

PACS number(s): 3.75.Fi, 3.65.Bz, 42.50.Vk, 42.50.Gy

Since the first experimental realisations of BoseEinstein condensation in dilute gases [1] 3], the properties and possible applications of condensates in various situations have been investigated. Most recently, attention has been drawn to the study of condensates in optical lattices [4 8] as have been intensely used in the context of laser cooling and trapping of clouds of noninteracting atoms [9]. But whereas the occupation of the lattice sites in an optical molasses for a cloud of laser cooled atoms at best is one atom per ten wells, the atomic densities found in a condensate allow for multiple occupation of each single well, which gives rise to a variety of new phenomena.

In this Letter we investigate the case of a condensate falling through a driven high-finesse optical cavity. The strong coupling of the condensed atoms to the cavity mode changes the resonance frequency of the cavity which hence is shifted into or out of resonance with the driving field. Consequently, the intracavity field intensity is modified and this can easily be measured by detecting the cavity output field. We show that according to the collective nature of the condensate this gives a measurable effect even for very low field intensities and for detunings from the atomic resonance frequency so large that the spontaneous scattering of photons is negligible. The proposed system should allow us to perform non-destructive measurements on the condensate. Similar systems have been used recently to predict amplification of matter waves [10] and the appearance of dressed condensates [11, 12].

Let us first introduce in more detail our model system, which is similar to that used recently to study the effect of a dynamically changing cavity field on the motion of a single atom 13 16.

We consider a Bose condensate consisting of $N$ two- level atoms of resonance frequency $\omega_{a}$ and spontaneous decay rate $\Gamma$ falling through an optical cavity. The atomcavity coupling is

$$
g(x, t)=g_{0} \cos (k x) e^{-\left(v_{z} t\right)^{2} /\left(2 w^{2}\right)},
$$

for a cavity mode in the form of a standing wave in the longitudinal direction and a Gaussian with waist $w$ transversally. The condensate is assumed to fall with constant velocity $v_{z}$, meaning that we neglect the transverse light forces on the atoms and the gravitational acceleration in the interaction region. Two further assumptions have been made at this point. First, the spatial extension of the condensate has to be small compared to the waist $w$ of the cavity in order to allow a quasi one-dimensional treatment. Second, we assume that the induced resonance frequency shift of the cavity [17,18 is much smaller than the longitudinal mode spacing, so that we can restrict the model to a single longitudinal mode with wavenumber $k$.

The cavity with resonance frequency $\omega_{c}$ and cavity decay rate $\kappa$ is externally driven by a laser of frequency $\omega$ with pump amplitude $\eta$ and is treated classically, that is, the intra-cavity field is described by a (complex) field amplitude $\alpha$.

As we are interested in the limit where the condensate is not destroyed by the light field, we will assume a large detuning $\Delta_{a}=\omega-\omega_{a} \gg \Gamma$ of the driving laser from the atomic resonance such that the saturation parameter $s=g_{0}^{2} / \Delta_{a}^{2} \ll 1$. Moreover, we want the cavity decay to dominate over the spontaneous decay of all atoms and thus impose the condition

$$
\kappa \gg N \Gamma s .
$$

In this realistically achievable limit we are not only allowed to adiabatically eliminate the excited state of the atoms but also to completely omit the effect of atomic decay.

Hence we obtain the equation of motion for the field amplitude

$$
\dot{\alpha}(t)=\left[i \Delta_{c}-i N\langle U(\hat{x}, t)\rangle-\kappa\right] \alpha(t)+\eta
$$

where $\Delta_{c}=\omega-\omega_{c}$ is the cavity-pump detuning, $U(x, t)=$ $g(x, t)^{2} / \Delta_{a}$ the optical potential per photon, and " $\langle\ldots\rangle$ " denotes the expectation value taken with respect to the condensate wave function $|\psi(t)\rangle$ at this time. This term describes the action of the condensate on the cavity field: the refractive index of the condensate shifts the resonance frequency of the cavity by an amount of $N\langle U(\hat{x}, t)\rangle$. 
(With very high finesse optical cavities this effect has already been observed even for a single atom [16,19, 20], that is, for $N=1$.) If we require that this effect significantly changes the intra-cavity field intensity in order to yield a measurable difference in the cavity output, then the maximum frequency shift must be of the order of or larger than the cavity line width $\kappa$, which implies

$$
N g_{0}^{2} / \Delta_{a} \geq \kappa
$$

From this we obtain an order of magnitude for the required detuning $\Delta_{a}$ which we insert into Eq. (2) to obtain the following condition for the cavity parameters:

$$
\frac{N g_{0}^{2}}{\Gamma \kappa} \gg 1
$$

The condensate wave function itself obeys a nonlinear Schrödinger equation (known as the Gross-Pitaevskii equation) with the Hamiltonian

$$
H=\frac{\hat{p}^{2}}{2 m}+|\alpha(t)|^{2} U(\hat{x}, t)+N g_{\text {coll }}|\psi(\hat{x}, t)|^{2}
$$

where the last term describes two-particle collisions between the condensed atoms and is related to the s-wave scattering length $a$ by $g_{\text {coll }}=4 \pi \hbar^{2} a / m$. This Hamiltonian, together with Eq. (3) for the cavity field, forms a set of coupled nonlinear equations describing the dynamics of the compound system formed by the condensate and the optical cavity.

In this Letter we will only consider the special case where the cavity decay rate $\kappa$ is much larger than the oscillation frequency of bound atoms in the optical potential of the cavity. In this limit the intra-cavity field amplitude follows adiabatically the condensate wavefunction and hence at any time is given by

$$
\alpha(t)=\frac{\eta}{\kappa-i\left[\Delta_{c}-N\langle U(\hat{x}, t)\rangle\right]} .
$$

Thus, the light intensity of the cavity output, which is proportional to $|\alpha|^{2}$, provides information about the condensate wavefunction. In the following we will investigate this effect in certain special parameter limits.

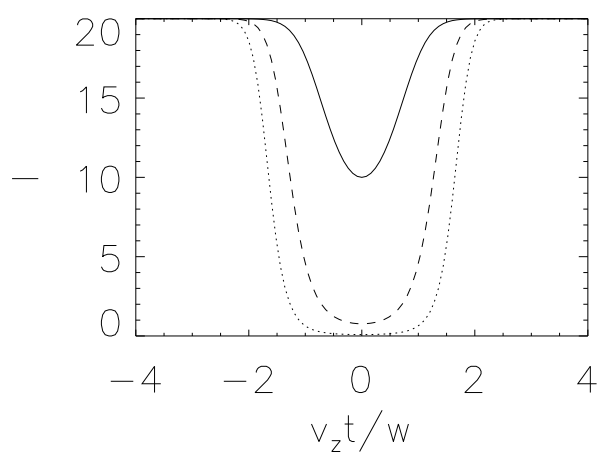

FIG. 1. Cavity photon number $I=|\alpha|^{2}$ versus time for a condensate falling through the cavity without being perturbed by the cavity field (see text for details). The optical potential depth is given by $N U_{0}=2 \kappa$ (solid line), $10 \kappa$ (dashed), and $30 \kappa$ (dotted), respectively. The pump field is chosen to give rise to 20 photons in the empty cavity and the detuning is $\Delta_{c}=0$.

Let us first consider the simple case where the cavity field is weak enough and the interaction time $\tau=w / v_{z}$ is short enough such that the condensate wavefunction remains essentially unperturbed (flat on the length scale of an optical wavelength). In this limit the cavity field can be evaluated analytically by inserting the frequency shift per atom

$$
\langle U(\hat{x}, t)\rangle=\frac{U_{0}}{2} e^{-\left(v_{z} t\right)^{2} / w^{2}}
$$

into Eq. (7), where we have introduced $U_{0}=g_{0}^{2} / \Delta_{a}$. In Fig. 1 the resulting mean cavity photon number $I=|\alpha|^{2}$ is plotted as a function of time for different atom numbers in the condensate or, equivalently, for different optical potentials.

For the parameters chosen in Fig. 1, the empty cavity is in resonance with the driving field but is shifted out of resonance by the presence of the condensate. Hence the condensate is detected by the absence of light, which further reduces the action of the cavity onto the condensate. Therefore the cavity provides a non-perturbative method of detection. The maximum resolution of the detection is limited, however, by the cavity waist $w$ and is not good enough to detect fine structures such as condensate interference fringes. It might be useful, however, to measure the output of an atom laser as has recently been realised experimentally [21.22]. Note also that this detection scheme only relies on the density of the condensate, not on its coherence, and thus in principle works as well with an incoherent cloud of atoms.

Let us now consider the opposite limit of a condensate falling very slowly through the cavity. We will find that under such conditions the condensate is adiabatically transfered into the lowest bound state of the optical potential and hence is strongly localized. In this case we must use the position and time dependent optical potential

$$
U(\hat{x}, t)=U_{0} \cos ^{2}(k \hat{x}) e^{-\left(v_{z} t\right)^{2} / w^{2}},
$$

so that the condensate at all times feels a periodic optical potential with periodicity $\lambda / 2$. The condensate wavefunction is conveniently described in terms of Bloch states

$$
\psi_{n, q}(x)=e^{i q x} \phi_{n, q}(x)
$$

where the functions $\phi_{n, q}, n \geq 0$, are periodic and the Bloch momentum $\hbar q$ is confined to the interval $[-\hbar k, \hbar k]$. Note that the coherent interaction of the condensate with the cavity light only couples Bloch states of different $n$ 
but leaves states of different $q$ decoupled and thus $q$ is a conserved quantity.

We are interested in the adiabatic limit corresponding to small transverse velocities $v_{z} \ll w / \tau_{R}$, where $\tau_{R}$ is the inverse of the recoil frequency $\omega_{R}=\hbar k^{2} /(2 m)$. In this limit the coherent time evolution associated with a potential of the form of Eq. (9) maps the Bloch energy bands $n$ onto the free space momentum intervals $[-(n+1) \hbar k,-n \hbar k]$ and $[n \hbar k,(n+1) \hbar k]$. This phenomenon is known and has been exploited in the context of laser cooling [23,24]. The same effect can be used in our model to transfer a falling condensate with a transverse momentum distribution confined in $[-\hbar k, \hbar k]$ into the lowest energy band $(n=0)$ of the optical potential inside the cavity. However, the situation is more complex here than for the case of laser cooling for two reasons. First, the light intensity itself depends on the condensate wavefunction and hence on time. Second, the condensate wavefunction obeys the nonlinear Schrödinger equation. Hence, for any given z-position of the condensate (any given time) the lowest energy state has to be found by self-consistently solving Eq. (7) and the Gross-Pitaevskii equation.
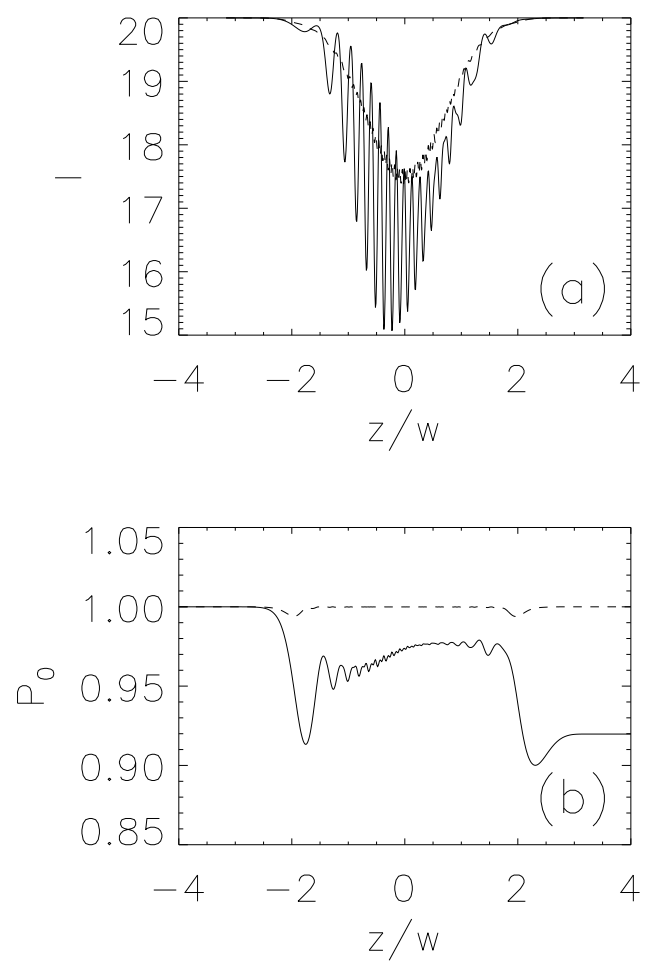

FIG. 2. Adiabatic transfer of the condensate wavefunction to the bound ground state. $g_{\text {coll }}=0, \Delta_{c}=0, N U_{0}=10 \kappa$, $v_{z}=w /\left(3 \tau_{R}\right)$ (dashed curves) respectively $v_{z}=w / \tau_{R}$ (solid curves). (a) Intra-cavity photon number $I=|\alpha|^{2}$, (b) population of instantaneous ground state corresponding to the photon number at any time. The initial condensate wavefunction is taken as the zero momentum eigenstate $|p=0\rangle$.

We show in Fig. 2 the time evolution of the intra-cavity field intensity $I$ and the overlap $P_{0}$ of the condensate wavefunction with the lowest energy band as the condensate is falling through the cavity without atomic collisions $\left(g_{\text {coll }}=0\right)$. For simplicity we assume that the initial condensate wavefunction is the free momentum state of zero momentum. The conservation of the Bloch momentum $q$ then ensures that at any time only the Bloch states with $q=0$ are populated and the overlap with the lowest energy band is given by

$$
P_{0}=\left|\left\langle\psi \mid \phi_{n=0, q=0}\right\rangle\right|^{2} .
$$

For a condensate falling with a velocity $v_{z}=w / \tau_{R}$ the transfer of the free wavefunction into the optical potential is not adiabatic (especially not on entering and leaving the cavity) and the occupation of the ground state drops to about $90 \%$. Hence there is a significant occupation of excited modes and the corresponding spatial oscillation of the condensate is reflected in the oscillation of the cavity output. For $v_{z}=w /\left(3 \tau_{R}\right)$, however, nearly all of the population is transfered into the lowest bound state and accordingly the oscillations are suppressed.

For the parameters of Fig. 2, $U_{0}>0$ and the condensate is attracted to the nodes of the light field. Hence, the lowest bound state is localized at these positions which leads to a much reduced coupling of the condensate to the cavity and correspondingly to a much smaller frequency shift of the cavity resonance. This can be easily seen by comparing the results for the cavity field with the solid line of Fig. 1., which is taken for the same parameters but a condensate falling so fast that the wavefunction does not have the time to change due to the presence of the optical potential wells.

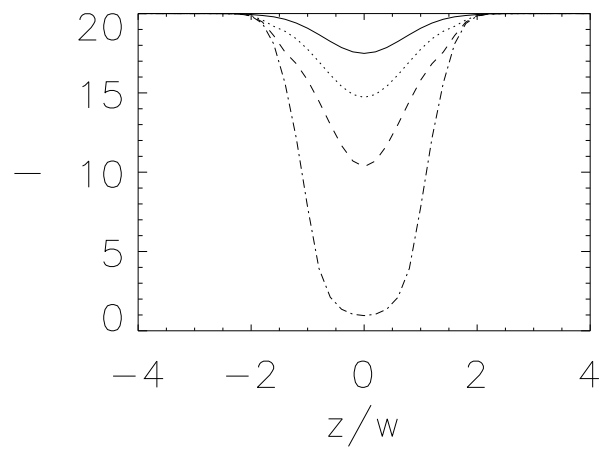

FIG. 3. Photon number corresponding to self-consistent ground state vs position of condensate in the cavity mode. $N g_{\text {coll }}=0,5,10,20 \omega_{R}$ (from top to bottom), $\Delta_{c}=0$, $N U_{0}=10 \kappa$. (For Rb and $\mathrm{Na}$ experiments, $N g_{\text {coll }}=\omega_{R}$ corresponds to condensate densities of the order of 30 atoms per potential well, i.e., per $(\lambda / 2)^{3}$.)

Figure 3 shows the effects of collisions on the intracavity photon number in the adiabatic regime $\left(v_{z} \ll w / \tau_{R}\right)$. The curves are derived from the self-consistent solution of Eq. (7) and the Gross-Pitaevskii equation for different values of $g_{\text {coll }}$. For increasing $g_{\text {coll }}$ the atoms in the condensate increasingly repell each other, counteracting the 
confining effect of the optical potential. The wavefunction becomes broader and the coupling of the condensate to the cavity stronger, which in turn leads to larger shifts in the cavity resonance frequency and a reduced cavity field intensity. Hence, the decrease in the cavity output provides a direct measure of the atom-atom interaction within the condensate. Note that this could be used for in situ measurements of Feshbach resonances, if one manipulates the s-wave scattering length by applying a magnetic field 25 28].

Finally, we plot in Fig. 1 the energy of the selfconsistent ground state and of the two lowest excitations. The latter are calculated in lowest order perturbation theory, that is, using the self-consistent ground state for the collisional term of the Hamiltonian (6). The energy difference between these states is the one which is responsible for the oscillation of the cavity output in the case of non-perfect adiabatic transfer of the condensate wavefunction into the lowest bound state as shown previously in Fig. 2.

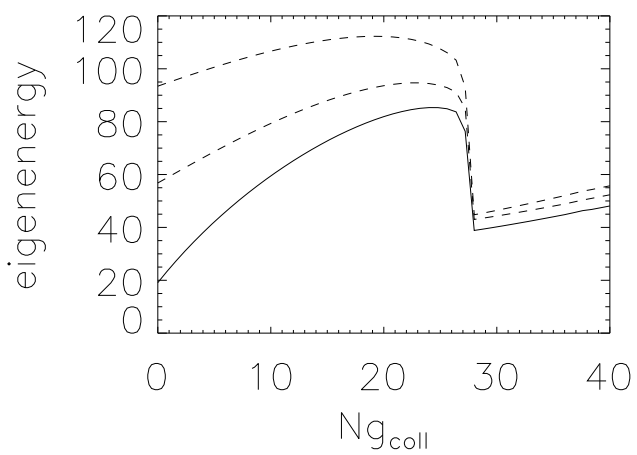

FIG. 4. Eigenenergy of the self-consistent ground state (solid curve) and of the first two condensate excitation states (dashed curves) versus collision parameter $g_{\text {coll }}$ for $\Delta_{c}=0$, $N U_{0}=10 \kappa$. Eigenenergies and $g_{\text {coll }}$ are given in units of $\omega_{R}$.

For $g_{\text {coll }}=0$ the ground state is strongly localized at the antinodes of the cavity leading to a large intracavity field intensity. Hence the lowest three eigenstates are well approximated by harmonic oscillator states and the frequency difference between the ground state and the first excited state is the same as the frequency difference between the first and the second excited state. For increasing $g_{\text {coll }}$ the internal (collisonal) energy of the condensate increases. Simultaneously the effective potential (optical potential plus collisional term) for the excited modes changes its shape from a harmonic oscillator to a potential well with a nearly flat bottom. This is due to the well-known fact that the ground state of the Gross-Pitaevskii equation becomes proportional to the negative confining potential if the collisional term dominates over the kinetic energy term in the Hamiltonian (Thomas-Fermi limit). Hence the excited modes are those of a potential which looks more like a box in this limit and the level spacing increases between more highly excited modes. However, as the wavefunction broadens with increasing $g_{\text {coll }}$ the cavity field intensity decreases and above a certain value $\left(N g_{\text {coll }} \approx 28 \omega_{R}\right.$ for the parameters in Fig. (1) no bound state exists in the optical potential. Hence the eigenfunctions above this critical value resemble free space momentum states which leads to the significant change in the behaviour of the spectrum of excitation energies.

In conclusion we have shown that a high-quality optical cavity provides a powerful tool to investigate properties of Bose-Einstein condensates in a non-destructive way. For slow initial velocities the condensate is adiabatically transfered into the self-consistent ground state of the optical potential, which contains ample information on condensate properties.

In addition, the system suggests many interesting applications. Using cavities with a decay rate $\kappa$ of the order of the condensate vibrational frequencies in the potential, the finite response time of the cavity implies a damping (or amplification) of the condensate oscillations as it has been shown for cooling and trapping of a single atom [13, 14, 16. By changing the intensity and/or the frequency of the driving laser depending on the cavity output one gets a handle for the controlled non-destructive in situ manipulation of the condensate wavefunction. Similarly, changing the magnitic field during the condensate passage should allow a direct measurement of the effective scattering length and the relaxation dynamics.

This work was supported by the United Kingdom EPSRC, the Austrian Science Foundation FWF (P13435), and the European Comission TMR network (FRMXCT96-0077).

[1] M. H. Anderson et al., Science 269, 198 (1995).

[2] C. C. Bradley et al., Phys. Rev. Lett. 75, 1687 (1995).

[3] K. B. Davies et al., Phys. Rev. Lett. 75, 3969 (1995).

[4] M. Kozuma et al., Phys. Rev. Lett. 82, 871 (1999).

[5] D.-I. Choi and Q. Niu, Phys. Rev. Lett. 82, 2022 (1999).

[6] D. Jaksch et al., Phys. Rev. Lett. 81, 3108 (1998).

[7] K. Berg-Sørensen and K. Mølmer, Phys. Rev. A 58, 1480 (1998).

[8] K.-P. Marzlin and W. Zhang, Phys. Rev. A 59, 2982 (1999).

[9] S. Chu, C. Cohen-Tannoudji, W. D. Phillips, Nobel lectures, Rev. Mod. Phys. 70, 685-742 (1998).

[10] C. K. Law and N. P. Bigelow, Phys. Rev. A 58, 4791 (1998).

[11] E. V. Goldstein, E. M. Wright, and P. Meystre, Phys. Rev. A 57, 1223 (1998).

[12] M. G. Moore and P. Meystre, Phys. Rev. A 59, R1754 (1999); M. G. Moore, O. Zobay, and P. Meystre, e-print cond-mat/9902293.

[13] P. Horak et al., Phys. Rev. Lett. 79, 4974 (1997).

[14] G. Hechenblaikner et al., Phys. Rev. A 58, 3030 (1998).

[15] A. C. Doherty et al., Phys. Rev. A 56, 833 (1997).

[16] P. Münstermann et al., Phys. Rev. Lett. 82, 3791 (1999).

[17] A. S. Parkins and D. F. Walls, Phys. Rep. 303, 1 (1998). 
[18] L. V. Hau et al., Nature 397, 594 (1999).

[19] H. Mabuchi et al., Opt. Lett. 21, 1393 (1996); C. J. Hood et al., Phys. Rev. Lett. 80, 4157 (1998).

[20] P. Münstermann et al., Opt. Commun. 159, 63 (1999).

[21] M.-O. Mewes et al., Phys. Rev. Lett. 78, 582 (1997).

[22] I. Bloch, T. W. Hänsch, and T. Esslinger, Phys. Rev. Lett. 82, 3008 (1999).

[23] A. Kastberg et al., Phys. Rev. Lett. 74, 1542 (1995).

[24] H. Stecher et al., Phys. Rev. A 55, 545 (1997).

[25] S. Inouye et al., Nature 392, 151 (1998).

[26] P. Courteille et al., Phys. Rev. Lett. 81, 69 (1998).

[27] E. Tiesinga, B. J. Verhaar, and H. T. C. Stoof, Phys. Rev. A 47, 4114 (1993).

[28] Y. Kagan, E. L. Surkov, and G. V. Shlyapnikov, Phys. Rev. Lett. 79, 2604 (1997). 